\title{
Gait identification using one-vs-one classifier model
}

\begin{abstract}
Gait has been used in many research area including medical and health. One of the ways to capture gait signal is by using the accelerometer sensor in the smartphone. In this work, gait signal is used to identify a person. The accuracy of the gait recognition while the phone held in the palm is evaluated. Besides that, the factor of linear interpolation is examined. Lastly, kNN, MLP and SVM algorithm are compared in determining the best accuracy that works best with the OvO classifier model. From the experiment, it can be seen that the gained accuracy for k-NN and MLP are both $96.7 \%$ with only 1 misclassified. Although the work is not related to medical and health, somehow it could provide the basis in healthcare related application. From the result, it is possible in adopting the proposed method in classifying decision based on the gait signal for medical and health purposes.
\end{abstract}

Keyword: Gait; K-NN; MLP; OvO; SVM 